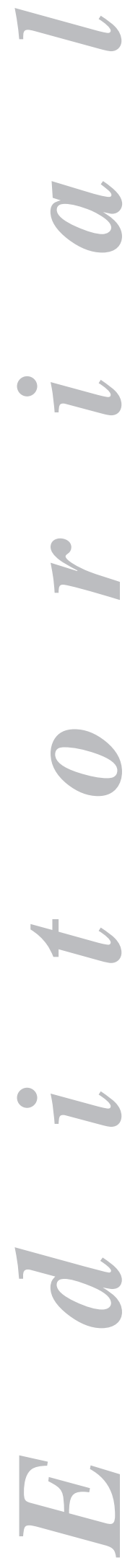

\section{Viral dynamics and prediction of response to treatment with pegylated interferon and ribavirin in patients with chronic hepatitis $\mathrm{C}$}

Chronic infection with hepatitis $\mathrm{C}$ virus (HCV) affects a huge number of people worldwide and may progress to liver cirrhosis and hepatocarcinoma. It is the first cause of liver transplantation in western countries, and results in major healthcare and social costs. Treatment with pegylated interferon (PEG-IFN) and ribavirin (RBV) may modify disease progression if successful in eradicating infection, which only occurs in slightly more than half of patients. The rest, around $40 \%$, will only receive a costly medication that usually impairs quality of life and may at times induce serious adverse events with no benefits whatsoever. Such events increase in number in the presence of concomitant medication including erythropoietin or colony-stimulating factors, which allow initiating or maintaining therapy in cytopenic subjects. A significant number of infected patients who would potentially benefit from therapy remain untreated because of such uncertainty and likely general status worsening long-term, and PEG-IFN + RBV is commonly administered only to subjects with advanced disease, where effectiveness is lower. Such circumstances have encouraged a search for response predictors, either negative or positive.

Male gender, age older than 45 years, and overweight are known to be associated with lower response rates. However, none of these factors, or their simultaneous occurrence, will determine therapy failure; nor their absence accurately predicts therapy success. When other parameters are also considered, including genotype, viral load, body mass index, liver fibrosis extent, alcohol use or iron metabolism, predictive capabilities improve, but a relevant group of patients remains where treatment results in unexpected effects. Only when two extreme groups are considered, with hypothetical cohorts (1) where each and every patient has all favorable or unfavorable parameters (body mass index above 30 $\mathrm{kg} / \mathrm{m}^{2}$ or below $20 \mathrm{~kg} / \mathrm{m}^{2}$; age older than 60 years or younger than 20 ; viral load greater than $9,000,000 \mathrm{IU} / \mathrm{ml}$ or smaller than $40,000 \mathrm{IU} / \mathrm{ml}$; advanced or absent liver fibrosis; binge or no drinking, abnormal or normal transaminase index), are significant differences observed. However, such hypothetical cohorts are far removed from the real scenarios where treatment decisions are to be made.

The contribution of other parameters requiring biochemical tests unusual in daily practice has been investigated - increased peripheral insulin resistance is associated with lower chances of response to PEG-INF + RBV, but more than $30 \%$ of patients with HOMA $>2$ will eventually exhibit virus eradication (2). Quasispecies present before treatment onset influence such response (3).

Evidence that a number of host-related factors, in addition to virus-related factors, condition treatment response has prompted genetic studies initially on the major histocompatibility complex (4) and then on numerous genes in a quest 
for a genetic signature predictive of response (5). Research is ongoing using powerful technologies to broadly analyze the human genome (6).

An analysis of data obtained for the accreditation of therapy with PEG-IFNo2a $+\mathrm{RBV}$ was first to provide a factor with a high negative predictive value: when viral load was not negative or had at least decreased by $2 \log _{10}$ after 12 weeks on treatment (early virological response), a sustained response could only be expected in $3 \%$ of treated individuals. Using PEG-IFN $\alpha 2 b+\mathrm{RBV}$, the odds for response under those same conditions was $0 \%$. The importance of these results is greater, inasmuch as they refer to carriers of viral genotype 1, which induces a lower response rate and requires treatment for 48 weeks. Failure to respond early has led to therapy discontinuation after 3 months in patients who otherwise would have received medication for longer periods of time without ever reaching viral eradication; however, a sustained response cannot be taken for granted in positive cases, and will only be found in around $70 \%$ of subjects $(7,8)$.

The study of viral kinetics during therapy allowed to identify a so-called rapid response (when viral load as assessed with a sensitive method is negative after 4 weeks), and also led to predict response at the end of treatment (9-12); it also allowed to variably shorten length of therapy in patients infected with any genotype (13-15), and to suggest prolonged therapy for those infected with genotype 1 in the absence of rapid response (16).

While the identification of such response types - given their negative predictive value - has allowed to reduce discomfort, adverse effects, and costs by discontinuing treatment when advisable, it also served to encourage physicians and patients to improve adherence when there is a response, and to initiate therapy early in order to rapidly have reliable predictive information available. Several studies suggest that decreases in viral load will be identified in association with potential sustained responses in much less than 4 weeks, maybe during the first few days on therapy (17-19). In the current issue of Revista Española de Enfermedades Digestivas, Hernández et al. (20) deal with this issue by researching the relationship between viral load decrease within one month after treatment onset and the possibility of predicting response at therapy completion; they find a high predictive value for rapid response, and also for reductions by $2 \log _{10}$ at 2 and 4 weeks, despite the series' limited size. This amount of viral load reduction leads to consider rapid response at weeks 2 and 4, and if the expected response at 3 months ultimately occurs much earlier this will logically identify those who will eventually have their infection eradicated (provided they will complete therapy), even though the negative predictive value becomes necessarily lower, since patients with smaller viral load decreases may later present with early response and then exhibit sustained response. This paper suggests that a viral dynamics approach may offer still more information regarding the prediction of PEG-IFN + RBV effectiveness in patients with chronic infection with HCV.

Sustained response, or a lack thereof, may be considered the result and expression of a number of forces that, on the one hand, facilitate and, on the other hand, oppose virus eradication. Such forces include all the aforementioned factors and them some unknown ones, and essentially refer to viral sensitivity and capacity to block therapy-induced mechanisms and immune response in each specific patient. This interpretation may account for the predictive ability of both early and rapid responses (as an expression of the balance between forces during early treatment), which is higher than that obtained when considering the limited number of factors involved in sustained response. 


\section{Emeritus, Servicio Andaluz de Salud. Hospitales Universitarios Virgen del Rocío.} Sevilla, Spain

\section{REFERENCES}

1. Foster GR, Fried MW, Hadziyannis SJ, Messinger D, Freivogel K, Weiland O. Prediction of sustained virological response in chronic hepatitis $C$ patients treated with peginterferon alfa-2a (40KD) and ribavirin. Scand J Gastroenterol 2007; 42: 247-55.

2. Romero-Gómez M, del Mar Viloria M, Andrade RJ, Salmerón J, Diago M, Fernández-Rodríguez CM. Insulin resistance impairs sustained response rate to peginterferon plus ribavirin in chronic hepatitis $\mathrm{C}$ patients. Gastroenterology 2005; 128: 636-41.

3. Salmerón J, de Rueda PM, Ruiz-Extremera A, Casado J, Huertas C, Bernal MC, et al. Quasispecies as predictive response factors for antiviral treatment in patients with chronic hepatitis C. Dig Dis Sci 2006; 51: 9607.

4. Romero-Gómez M, González-Escribano MF, Torres B, Barroso N, Montes-Cano MA, Sánchez-Muñoz D, et al. HLA class I B44 is associated with sustained response to interferon + ribavirin therapy in patients with chronic hepatitis C. Am J Gastroenterol 2003; 98: 1621-6.

5. Asselah T, Bieche I, Narguet S, Sabbagh A, Laurendeau I, Ripault MP, et al. Liver gene expression signature to predict response to pegylated interferon plus ribavirin combination therapy in patients with chronic hepatitis C. Gut 2008; 57: 516-24.

6. Aurora R, Donlin MJ, Cannon NA, Tavis JE. Genome-wide hepatitis C virus amino acid covariance networks can predict response to antiviral therapy in humans. J Clin Invest 2009; 119: 225-36.

7. Fried MW, Shiffman ML, Reddy KR, Smith C, Marinos G, Gonçales FL, Jr., et al. Peginterferon alfa-2a plus ribavirin for chronic hepatitis C virus infection. N Engl J Med 2002; 347: 975-82.

8. Davis GL, Wong JB, McHutchison JG, Manns MP, Harvey J, Albrecht J. Early virologic response to treatment with peginterferon alfa- $2 \mathrm{~b}$ plus ribavirin in patients with chronic hepatitis C. Hepatology 2003; 38: 64552.

9. Ferenci P. Predicting the therapeutic response in patients with chronic hepatitis $\mathrm{C}$ : the role of viral kinetic studies. J Antimicrobial Chemotherapy 2004; 53: 15-8.

10. Bárcena R, Moreno A, del Campo S, Muriel A, Mateos ML, Garrido E, et al. The magnitude of week 4 HCV RNA decay on pegylated interferon/ribavirin accurately predicts virological failure in patients with genotype 1. Antivir Ther 2007; 12: 401-6.

11. Jensen DM, Morgan TR, Marcellin P, Pockros PJ, Reddy KR, Hadziyannis SJ, et al. Early identification of $\mathrm{HCV}$ genotype 1 patients responding to 24 weeks peginterferon alpha-2a $(40 \mathrm{kd}) /$ ribavirin therapy. Hepato$\operatorname{logy} 2006$; 43: 954-60.

12. Yu JW, Wang GQ, Sun LJ, Li XG, Li SC. Predictive value of rapid virological response and early virological response on sustained virological response in $\mathrm{HCV}$ patients treated with pegylated interferon alpha- $2 \mathrm{a}$ and ribavirin. J Gastroenterol Hepatol 2007; 22: 832-6.

13. Mangia A, Santoro R, Minerva N, Ricci GL, Carretta V, Persico M, et al. Peginterferon alfa-2b and ribavirin for 12 vs. 24 weeks in HCV genotype 2 or 3. N Engl J Med 2005; 352: 2609-17.

14. Ferenci P, Fried MW, Shiffman ML, Smith CI, Marinos G, Gonçales FL, Jr., et al. Predicting sustained virological responses in chronic hepatitis $\mathrm{C}$ patients treated with peginterferon alfa- $2 \mathrm{a}(40 \mathrm{KD}) /$ ribavirin. J Hepatol $2005 ; 43$ : 425-33.

15. Zeuzem S, Buti M, Ferenci P, Sperl J, Horsmans Y, Cianciara J. Efficacy of 24 weeks treatment with peginterferon alfa- $2 \mathrm{~b}$ plus ribavirin in patients with chronic hepatitis $\mathrm{C}$ infected with genotype 1 and low pretreatment viremia. Gastroenterology 2006; 131: 451-60.

16. Sánchez-Tapias JM, Diago M, Escartín P, Enríquez J, Romero-Gómez M, Bárcena R, et al. Peginterferonalfa2a plus ribavirin for 48 versus 72 weeks in patients with detectable hepatitis C virus RNA at week 4 of treatment. Gastroenterology 2006; 131: 451-60.

17. Layden JE, Layden TJ, Reddy KR, Levy-Drummer RS, Poulakos J, Neumann AU. First phase viral kinetic parameters as predictors of treatment response and their influence on the second phase viral decline. J Viral Hepat 2002; 9: 340-5.

18. Hsu CS, Liu CJ, Lai MY, Chen PJ, Kao JH, Chen DS. Early viral kinetics during treatment of chronic hepatitis $C$ virus infection with pegylated interferon alpha plus ribavirin in Taiwan. Intervirology 2007; 50: 310-5.

19. Buti M, Sánchez-Avila F, Lurie Y, Stalgis C, Valdés A, Martell M, et al. Viral kinetics in genotype 1 chronic hepatitis $\mathrm{C}$ patients during therapy with 2 different doses of peginterferon alfa-2b plus ribavirin. Hepatology 2002; 35: 930-6.

20. Hernández A, Domper F, León A, Lorente R, López B, de la Santa E, et al. Viral kenitics during the first month of treatment in patients with genotype 1 chronic hepatitis C. Rev Esp Enferm Dig 2009; 101: 671-9. 


\section{Dinámica viral y predicción de la respuesta al tratamiento con interferón pegilado y ribavirina en pacientes con hepatitis crónica por virus $\mathbf{C}$}

La infección crónica por el virus de la hepatitis C (VHC) afecta a un gran número de personas en todo el mundo y puede evolucionar a cirrosis hepática y hepatocarcinoma. En los países occidentales es la primera causa de trasplante hepático y da lugar a costes sanitarios y sociales de importancia. El tratamiento con interferón pegilado (Peg IFN) y ribavirina (RBV) es capaz de modificar la evolución si consigue la erradicación de la infección, lo que tiene lugar sólo en algo más de la mitad de los pacientes tratados. El resto, alrededor del 40\%, recibe una medicación cara, que habitualmente empeora su calidad de vida y que, en ocasiones, puede provocar efectos adversos graves, sin que tenga utilidad alguna. Estos acontecimientos se ven incrementados si se decide administrar simultáneamente otros fármacos -eritropoyetina, factores estimulantes de colonias- que permitan iniciar o mantener el tratamiento en pacientes con citopenias. Un importante número de infectados que podrían beneficiarse quedan sin tratamiento debido a esta incertidumbre y a la perspectiva de empeorar su estado general durante un periodo prolongado de tiempo, y es frecuente que sólo reciban Peg IFN + RBV personas con signos evidentes de enfermedad avanzada, en los que la efectividad es menor. Estas circunstancias han animado a identificar factores predictivos de respuesta, positivos o negativos.

Es conocido que el sexo masculino, más de 45 años de edad y el sobrepeso, se asocian con menor tasa de respuesta. Sin embargo, ninguno de estos factores, y tampoco su presencia simultánea, asegura fallo del tratamiento; tampoco la ausencia de los tres predice con exactitud el éxito del mismo. Si, además, se tienen en cuenta otros parámetros -genotipo, carga viral, índice de masa corporal, grado de fibrosis hepática, consumo de alcohol metabolismo del hierro- la capacidad predictiva mejora, aunque sigue quedando un importante grupo de pacientes en los que el tratamiento tiene un efecto diferente al esperado. Sólo si se crean dos grupos extremos, configurando una cohorte hipotética (1) en la que cada paciente presente todos los valores favorables o desfavorables (índice de masa corporal superior a $30 \mathrm{~kg} / \mathrm{m}^{2} \mathrm{o}$ inferior a $20 \mathrm{~kg} / \mathrm{m}^{2}$; edad por encima de 60 años o no superior a 20; carga viral mayor de $9.000 .000 \mathrm{UI} / \mathrm{ml}$ o menor de $40.000 \mathrm{UI} / \mathrm{ml}$; fibrosis hepática avanzada o ausente; consumo de alcohol e índice de transaminasas) se obtienen diferencias muy valorables. Sin embargo, la cohorte hipotética está lejos de la realidad ante la que debe tomarse la decisión de tratar.

Se ha estudiado la relación de otros factores que requieren estudios bioquímicos no usuales en la práctica habitual: el aumento de resistencia periférica a la insulina se relaciona con menos posibilidades de respuesta a Peg+ RBV; pero más del $30 \%$ de los pacientes con HOMA > 2 terminan erradicando el virus (2); las cuasiespecies presentes antes de iniciar el tratamiento influyen en la respuesta al mismo (3).

La evidencia de que una serie de factores del huésped, además de los relativos al virus, condicionan la respuesta al tratamiento, ha dado lugar a estudios genéticos, empezando por el Complejo Mayor de Histocompatibilidad (4) y continuando por el 
análisis de numerosos genes hasta llegar a la búsqueda de una firma genética que asegure la respuesta (5). En este sentido continúan las investigaciones mediante potentes tecnologías capaces de analizar muy ampliamente el genoma (6).

El análisis de los datos obtenidos en el ensayo para la acreditación del tratamiento con Peg IFNo2a+ RBV permitió, por primera vez, conseguir un dato de alto valor predictivo negativo: si a las 12 semanas de recibir tratamiento la viremia no era negativa o no había descendido, al menos, $2 \log _{10}$-respuesta virológica precoz-, únicamente podría esperarse respuesta sostenida en el 3\% de los pacientes tratados (7). Utilizando Peg IFN $\alpha 2 b+\mathrm{RBV}$, la posibilidad de respuesta en las mismas condiciones fue el $0 \%$ (8). La importancia de estos resultados es mayor cuanto que se refieren a portadores del genotipo viral 1 , que induce menor tasa de respuesta y que precisa tratamiento durante 48 semanas. La falta de respuesta precoz ha servido para interrumpir el tratamiento a los tres meses a pacientes que, de otro modo, hubieran continuado recibiendo la medicación durante periodos más prolongados sin conseguir la erradicación de la infección; aunque en caso positivo no es posible asegurar que el paciente tendrá respuesta sostenida, ya que esta se producirá sólo en el 70\% aproximadamente de los casos $(7,8)$.

El estudio de la cinética viral durante el tratamiento ha permitido identificar la llamada respuesta rápida (cuando la viremia, determinada con una técnica sensible, es negativa a las 4 semanas) y ha servido para predecir la respuesta al final del tratamiento (9-12) y, también, para acortar la duración del mismo, en proporción variable, en los infectados por todos los genotipos (13-15), y también para proponer su prolongación en los infectados por genotipo 1 sin respuesta rápida (16).

Aunque la identificación de estos dos tipos de respuesta ha permitido -dado su valor predictivo negativo- reducir molestias, efectos adversos y costes suspendiendo el tratamiento cuando fuera aconsejable hacerlo, también ha servido para animar a médicos y pacientes a mejorar la adherencia cuando se produce respuesta, y a iniciar el tratamiento ante la perspectiva de disponer con rapidez de información predictiva fiable. Diversos estudios hacen suponer que podrán identificarse descensos de viremia relacionables con la posibilidad de obtener respuesta sostenida en plazos muy inferiores a 4 semanas, tal vez ya durante los primeros días del tratamiento (1719). En el presente número de la Revista Española de Enfermedades Digestivas, Hernández y cols. (20) se ocupan de este aspecto buscando la relación entre la cuantía del descenso de la carga viral antes del primer mes de tratamiento y la posibilidad de previsión de la respuesta al final de mismo, encontrando alto valor predictivo para la ya conocida respuesta rápida y, también, para descensos de $2 \log _{10}$ a las 2 y 4 semanas, a pesar de que la serie es relativamente corta. Esta intensidad del decremento de viremia supone adelantar el concepto de respuesta precoz a las semanas 2 y 4 , y es lógico que si la respuesta esperada a los 3 meses se produce mucho antes identifique a los que finalmente erradiquen la infección (siempre confiando en que completarán el tratamiento), aunque el valor predictivo negativo tenga que ser necesariamente menor, ya que pacientes con descensos de la carga viral inferiores a esa cifra pueden alcanzar con posterioridad respuesta precoz y terminar con respuesta sostenida. Este trabajo sugiere que la vía de la dinámica viral puede ofrecer todavía más información acerca de la predicción de la efectividad de Peg IFN + RBV en pacientes con infección crónica por VHC.

La respuesta sostenida, o su ausencia, puede interpretarse como el resultado y la expresión de una serie de fuerzas que, por una parte facilitan y por otra se oponen, a la erradicación del virus. Estas fuerzas están constituidas por todos los factores que se han citado y por otros desconocidos, y fundamentalmente se refieren a la sensibi- 
lidad del virus, a su capacidad para bloquear mecanismos inducidos por el tratamiento y a la respuesta inmune en cada paciente concreto. Esta interpretación puede explicar que la capacidad predictiva de las respuestas precoz y rápida -como expresión del balance de fuerzas ya en las primeras fases del tratamiento- sea mayor que la que se obtiene de la consideración de un número limitado de los factores que intervienen en la consecución de la respuesta sostenida.

J. Aguilar Reina

Emérito del Servicio Andaluz de Salud. Hospitales Universitarios Virgen del Rocío. Sevilla 